\title{
Monte Carlo model for studying the effects of melanin concentrations on retina light absorption
}

\author{
Ya Guo, ${ }^{1}$ Gang Yao, ${ }^{1, *}$ Bo Lei, ${ }^{2,3}$ and Jinglu Tan ${ }^{1}$ \\ ${ }^{1}$ Department of Biological Engineering, University of Missouri-Columbia, Columbia, Missouri 65211, USA \\ ${ }^{2}$ Department of Veterinary Medicine and Surgery, University of Missouri-Columbia, Columbia, Missouri 65211, USA \\ ${ }^{3}$ Department of Ophthalmology, University of Missouri-Columbia, Columbia, Missouri 65211, USA \\ *Corresponding author: yaog@missouri.edu
}

Received July 18, 2007; revised October 25, 2007; accepted November 20, 2007; posted November 28, 2007 (Doc. ID 85199); published January 10, 2008

\begin{abstract}
We developed a Monte Carlo model to calculate light absorption in human and mouse retinas. The retina was modeled as a five-layer spherical structure. The effects of melanin concentrations in the retinal pigment epithelium (RPE) and choroid layer were studied. Variations of blood content in choroid were also considered in the simulation. Our simulation results indicated that light absorption in neural retina was at least $20 \%$ higher in albino subjects than in pigmented subjects under both photobleaching and dark-adapted conditions. It can be four times higher at optical wavelengths corresponding to minimal hemoglobin absorption. The elevated absorption at neural retina was attributed to the light backscattered from the choroid and sclera layers. This simulation model may provide useful information in studying light-induced retina damage. () 2008 Optical Society of America

OCIS codes: $170.0170,170.3660,290.7050,330.7326$.
\end{abstract}

\section{INTRODUCTION}

Melanin, a dark-brown pigment abundantly present in the skin, hair, and eyes of many species, is one of the primary biological components for light absorption [1]. Melanin plays an important role in retina development and in maintaining normal retinal function and structure. It helps maintain visual acuity by absorbing the light passing through the retina and blocking backscattered light from the choroid and the sclera. Similar to the huge variations in skin color, melanin concentrations vary over a wide range among individuals. In the extreme case of albinism, melanin concentration is significantly reduced, which leads to some interesting ophthalmic phenomena [2]. For example, it is well known that albinos are vulnerable to light damage and are linked with photophobia and poor vision [3].

Electroretinogram (ERG) is commonly used to assess retinal function in both humans and animals. Previous studies in young children have indicated that albino subjects had stronger and faster ERG signals than normal pigmented subjects [4]. Similar results were obtained in small animal [5]. However, adult albino mice showed reduced ERG responses because of the loss of photoreceptors as well as a drop in the overall amount of rhodopsin [6]. Such a phenomenon was conjectured as a result of the higher light absorption in albino retina caused by reduced melanin concentrations. Chronic high light absorption can lead to retina damage.

Because retinal pigments, especially melanin, play important roles in light absorption, a quantitative study of melanin effects on light absorption in the retina can benefit our understanding of photon-induced retinal damage. Monte Carlo simulation has been used extensively in laser-tissue interaction studies and is considered as the most accurate [7] and versatile [8] method to study light propagation in biological tissues. It has been applied previously to calculate ocular spectral reflectance $[9,10]$. Hammer et al. [9] applied an inverse Monte Carlo method with a double-integrating-sphere technique to extract the optical parameters of each fundus layer. The measured optical properties were then used in a forward Monte Carlo method to predict human ocular fundus reflectance. Preece and Claridge [10] used the Monte Carlo method to simulate human ocular fundus reflectance under different melanin concentrations. Slab retinal models were often used in these studies, although their accuracy has not been investigated.

In this study, we used a spherical retina model in a Monte Carlo simulation to study the effects of retinal melanin concentrations on light absorption. Our results indicated that the slab retinal model can produce similar results as the spherical model in subjects with normal melanin concentrations. However, the slab model generated substantial errors in albino subjects. In addition, the spherical model revealed that eye size played an important role in affecting light absorption. Such size effects induced different light absorption features in human and mouse retinas.

\section{METHODS}

A. Retina Model

We modeled the retina as a spherical construct with five concentric sphere layers (Fig. 1): neural retina layer, retinal pigment epithelium (RPE), choroid, sclera, and semiinfinite thick-tissue layer outside the sclera. Light was incident upon the retina through the transparent ocular medium. The incident light was distributed uniformly 


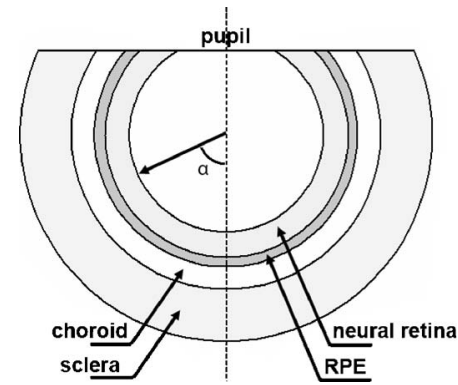

Fig. 1. Five-layer ocular fundus structure used in the simulation. The fifth layer was modeled as a semi-infinite medium outside the sclera.

across the retina layer within an azimuth angle of $60^{\circ}(\alpha$ in Fig. 1). At each point of the retinal surface, the incident angular profile followed the Lambertian distribution; i.e., the incident intensity was proportional to the cosine of the incident angle. The ocular diameters were $24.0 \mathrm{~mm}$ and $3.0 \mathrm{~mm}$ [11] in human and mouse retinas, respectively. The pupil diameter of the human retina was set as $7 \mathrm{~mm}$, and the pupil size of the mouse retina was set as $2 \mathrm{~mm}[12]$.

\section{B. Monte Carlo Method}

The basic Monte Carlo simulation process has been reported in detail elsewhere $[13,14]$. The photons were scattered and absorbed while propagating inside the tissue. The movement of a photon packet was traced using a sampling theory based on local scattering and absorption coefficients [7]. The Heyney-Greenstein phase function [15] was used to determine the scattering angles. At the interfaces of two layers with different refractive indices, light was reflected or transmitted to new directions that can be calculated as

$$
\begin{aligned}
& \mathbf{R}=\mathbf{I}+2 \mathbf{N} \cos \theta_{i}, \\
& \mathbf{T}=\frac{n_{1}}{n_{2}} \mathbf{I}-\left(\cos \theta_{t}-\frac{n_{1}}{n_{2}} \cos \theta_{i}\right) \mathbf{N},
\end{aligned}
$$

where $\mathbf{R}$ is the reflected vector; $\mathbf{T}$ is the transmitted vector; $\mathbf{I}$ is the incident vector; $\mathbf{N}$ is the normal vector at the intersection point; $n_{1}$ and $n_{2}$ are the refractive indices of the incident layer and the transmission layer, respectively; $\theta_{i}$ is the incident angle formed by $\mathbf{I}$ and $\mathbf{N}$; and $\theta_{t}$ is the refraction angle and satisfies Snell's law: $n_{1} \sin \theta_{i}$ $=n_{2} \sin \theta_{t}$.

When photons exit from the neural retina layer into the vitreous cavity, they may reenter the retina layer depending on the specific exit angle. The tracing of a specific photon packet ended with two conditions. First, the photon was completely absorbed inside the tissue. Second, the photon exited from the pupil of a predefined size. Those photons absorbed at each layer were recorded as the percentage absorption of total incident photons. Specifically, the absorption at the neural retina layer was analyzed in detail in this study.

\section{Optical Properties of Retina}

Each of the five spherical layers was characterized by a set of optical parameters, including absorption coefficient $\mu_{a}$, scattering coefficient $\mu_{s}$, refractive index $n$, and anisotropy $g$. Absorption coefficient $\mu_{a}$ refers to the probability of a photon being absorbed per unit of infinitesimal path length. Scattering coefficient $\mu_{s}$ is defined as the probability of a photon being scattered per unit of infinitesimal path length. The anisotropy $g$ is the mean cosine of the scattering angles $[7,10]$. Due to the lack of published experimental measurements on mouse retina, we assumed that the basic optical absorption and scattering coefficients of retina were the same in both normal human and wild-type mice. Consequently, the simulation results may not be numerically accurate for mice but should be meaningful for comparison purposes. The outermost (fifth) layer was modeled as an infinite environment with a typical tissue absorption coefficient of $0.1 \mathrm{~cm}^{-1}$, a scattering coefficient of $100 \mathrm{~cm}^{-1}$, and an anisotropy coefficient of 0.9 [16].

The geometry parameters, refractive indices, and scattering coefficients (Table 1) of each layer were obtained from previously published data $[9,10,17-21]$, which were found to be in good agreement with experimental observations $[9,10]$. Fresnel reflections between the boundaries of each of the four retina layers were neglected because little difference in the refractive index has been reported for posterior fundus tissue [10]. The refractive indices of the neural retina layer, RPE, choroid, and sclera layers were all $1.47[9,17]$. The refractive indices of the media below and above the four layers were 1.336 and 1.36 , respectively.

\begin{tabular}{|c|c|c|c|c|c|c|}
\hline \multirow[b]{2}{*}{ Tissue } & \multirow[b]{2}{*}{$n$} & \multirow[b]{2}{*}{$\begin{array}{c}\mu_{a}\left(\mathrm{~cm}^{-1}\right) \\
(\text { at } 510 \mathrm{~nm})\end{array}$} & \multirow{2}{*}{$\begin{array}{c}\mu_{s}\left(\mathrm{~cm}^{-1}\right) \\
(\text { at } 510 \mathrm{~nm})\end{array}$} & \multirow[b]{2}{*}{$g$} & \multicolumn{2}{|c|}{ Thickness $(\mu \mathrm{m})$} \\
\hline & & & & & Human & Mouse \\
\hline Vitreous and ILM $^{a}$ & 1.336 & - & - & - & - & - \\
\hline Neural retina & 1.47 & 3.7 & 87.5 & 0.97 & 200 & 220 \\
\hline RPE & 1.47 & $5408^{b}$ & 1142 & 0.84 & 10 & 8 \\
\hline Choroid & 1.47 & $1352^{b}$ & 613 & 0.87 & 250 & 23 \\
\hline Sclera & 1.47 & 4.9 & 1086 & 0.9 & 700 & 30 \\
\hline $\begin{array}{c}\text { Tissue } \\
\text { Background }\end{array}$ & 1.36 & 0.1 & 100 & 0.9 & $+\infty$ & $+\infty$ \\
\hline
\end{tabular}

Table 1. Refractive Indices and Optical Parameters of Human and Mouse Fundus

${ }^{a} \mathrm{ILM}$, inner limiting membrane.

${ }^{b} \mathrm{RPE}$ melanin; $4 \mathrm{~mol} / \mathrm{l}$; choroid melanin, $1 \mathrm{~mol} / \mathrm{l}$, choroid hemoglobin, $1.7 \mathrm{mmol} / 1$. 
The absorption coefficients of retina and sclera were obtained from previous studies [9]. However, it is worth pointing out that the retina absorption data were measured under bleaching conditions so that there was little contribution from photoreceptors. The absorption from photoreceptors needs to be included in order to investigate light absorption under normal light conditions. In this project, we first simulated the photobleached retina and then studied the dark-adapted retina in Subsection 3.E. The absorption coefficient of RPE is determined by its melanin concentration [22]:

$$
\mu_{a}^{R P E}=2.3 c_{m}^{R P E} \varepsilon_{m}(\lambda)
$$

where $c_{m}^{R P E}(\mathrm{~mol} / \mathrm{l})$ is the concentration of melanin in the RPE layer and $\varepsilon_{m}(\lambda)\left[\mathrm{cm}^{-1 /(\mathrm{mol} / \mathrm{l})}\right]$ is the molar extinction coefficient of melanin at wavelength $\lambda$. Optical properties are defined based on a natural logarithm calculation, while molar extinction coefficients are defined with a base-10 logarithm. Therefore the coefficient $\ln (10)$ (about 2.3) was introduced for such a unit conversion [23].

The absorption coefficient of choroid was determined by its melanin concentration and hemoglobin concentration:

$$
\mu_{a}^{\text {Choroid }}=2.3 c_{m}^{\text {Choroid }} \varepsilon_{m}(\lambda)+2.3 c_{b} \varepsilon_{b}(\lambda)
$$

where $c_{m}^{\text {Choroid }}$ is the concentration of melanin in the choroid layer, $\mathrm{c}_{b}(\mathrm{~mol} / \mathrm{l})$ is the blood concentration, and $\varepsilon_{b}(\lambda)$ $\left[\mathrm{cm}^{-1} /(\mathrm{mol} / \mathrm{l})\right]$ is the molar extinction coefficient of blood at wavelength $\lambda$. We assumed a $95 \%$ oxygenation ratio in blood, i.e., 95\% oxyhemoglobin and 5\% deoxyhemoglobin.

The molar extinction coefficients of melanin and hemoglobin at visible wavelengths have been compiled by Jacques [23]. The hemoglobin concentration was $1.7 \pm 1.49 \mathrm{mmol} / \mathrm{l}$ at the macula area and $1.73 \pm 1.80 \mathrm{mmol} / \mathrm{l}$ outside the macula area [14]. In our study, three different hemoglobin concentrations of $0.85 \mathrm{mmol} / \mathrm{l}, 1.7 \mathrm{mmol} / \mathrm{l}$, and $3.4 \mathrm{mmol} / \mathrm{l}$ were used.

Existing literature indicates that melanin content in the mouse retina varies from 0 to $500 \mu \mathrm{g}$ [24]. In our simulation, the melanin content in the retina of pigmented mice was set as $300 \mu \mathrm{g}$, which corresponded to $1.685 \mu \mathrm{mol}(178 \mathrm{~g} / \mathrm{mol})$. The diameter of the mouse eye was about $3 \mathrm{~mm}$ [11], the total thickness of the RPE was about $0.008 \mathrm{~mm}$ [18], and the thickness of the choroid layer was $0.023 \mathrm{~mm}$ [19] (refer to Table 1). In normal eyes, the melanin concentration in the RPE layer was about 4 times of that in choroid [10]. Based on the above information, we used $4.0 \mathrm{~mol} / \mathrm{l}$ and $1.0 \mathrm{~mol} / \mathrm{l}$ as melanin concentrations in RPE and choroid of a normal human, respectively. In albino subjects, the melanin concentration was very small and close to zero [24,25]. A multiplication factor from 0 to 1 was used in our studies to represent the melanin concentration variations in different subjects. This factor was referred to as the relative melanin concentration. The relative melanin concentration was 1.0 in pigmented retina and 0 in albino retina.

A total of $8 \times 10^{5}$ photons of a given wavelength were used in the simulation when the relative melanin concentration was smaller than 0.5 , and $3.2 \times 10^{6}$ photons were used when the relative melanin concentration was larger

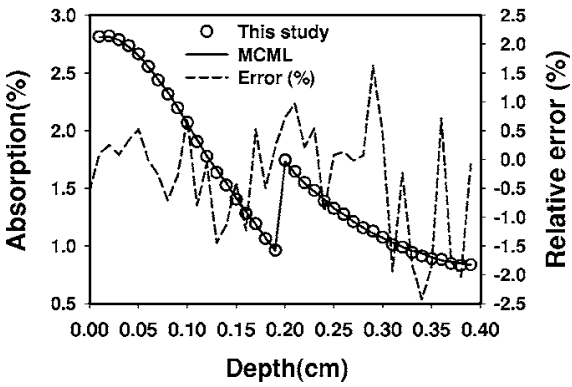

Fig. 2. Comparison between the spherical model used in this study and the MCML in the slab geometry. The optical properties of the three-layer sample are listed in Table 2.

than 0.5. These photon numbers ensured that the standard deviation of our simulation results was less than $0.1 \%$ of the mean value.

\section{RESULTS AND DISCUSSION}

\section{A. Program Validation}

In order to verify our program, we compared simulation results from our program with that from a standard Monte Carlo program (MCML) developed by Wang et al. [7]. Because the MCML was designed for a flat layer geometry, we used a very large radius in our spherical models to make it behave like a flat model. We found that the two programs generated the same results under different sample parameters. For example, Fig. 2 shows the simulated sample absorption at different depths inside a three-layer slab tissue that was used before as an example in the MCML manual. The optical properties of this sample are listed in Table 2. As expected, for slab tissues, the results calculated from our model and from MCML were nearly identical and the difference between them was less than $2.5 \%$. The small oscillatory residual errors were due mainly to the statistical nature of the Monte Carlo method.

\section{B. Effect of Spherical Geometry}

Because the spherical geometry adds a certain complexity in the computation compared to the conventional slab model, it is necessary to determine whether the spherical morphology of retina is important for computing retina absorption. We compared the results obtained from a flat retina model to those from our spherical model. As shown in Fig. 3, there was little difference in light absorption in human neural retina at normal melanin concentrations. However, the slab model significantly underestimated retina absorption in albino human eyes. In the albino hu-

Table 2. Parameters of a Three-Layer Tissue Model Used for Program Verification

\begin{tabular}{cccccc}
\hline Layer & $n$ & $g$ & $\begin{array}{c}\mu_{a} \\
\left(\mathrm{~cm}^{-1}\right)\end{array}$ & $\begin{array}{c}\mu_{s} \\
\left(\mathrm{~cm}^{-1}\right)\end{array}$ & $\begin{array}{c}\text { Thickness } \\
(\mathrm{cm})\end{array}$ \\
\hline Layer above & 1.0 & - & - & - & - \\
Layer 1 & 1.37 & 0.9 & 1 & 100 & 0.1 \\
Layer 2 & 1.37 & 0.0 & 1 & 10 & 0.1 \\
Layer 3 & 1.37 & 0.7 & 2 & 10 & 0.2 \\
Layer below & 1.0 & - & - & - & - \\
\hline
\end{tabular}




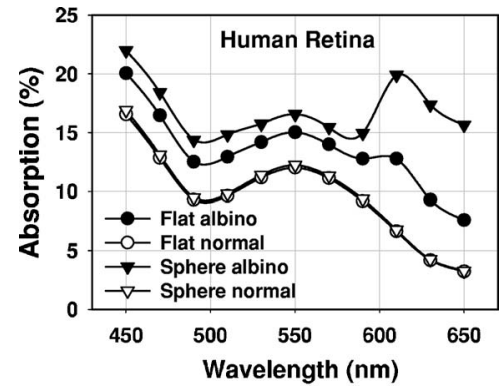

Fig. 3. Comparison between the spherical and the slab retina models in bleached human neural retina. The hemoglobin concentration used in the simulation was $1.7 \mathrm{mmol} / \mathrm{l}$.

man retina layer, the percentage difference between the slab model and our spherical model was $10 \%-17 \%$ in the bandwidth of 450 to $590 \mathrm{~nm}$ and $56 \%-101 \%$ in the red wavelength region $(>600 \mathrm{~nm})$.

\section{Retina Absorption at Different Melanin Concentrations}

Figure 4 shows the effects of melanin concentration on optical absorption in the human neural retina layer at three different choroid hemoglobin concentrations: $0.85,1.7$, and $3.4 \mathrm{mmol} / \mathrm{l}$. Results shown were obtained at three wavelengths: $510 \mathrm{~nm}, 550 \mathrm{~nm}$, and $630 \mathrm{~nm}$. The normal melanin level in human retina depends on race or skin color. At visible wavelengths, the retinal absorption varied little when the relative melanin concentration changed from 0.2 to 1.0 . These results implied that such normal variations in melanin concentration have a mini-
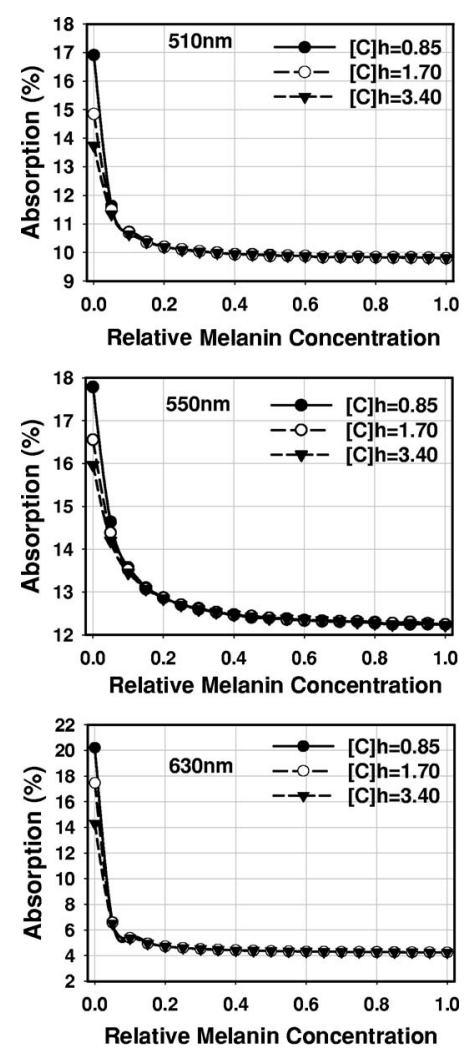

Fig. 4. Effects of melanin concentration on light absorption in bleached human neural retina at three different wavelengths $(510 \mathrm{~nm}, 550 \mathrm{~nm}$, and $630 \mathrm{~nm})$. mal effect on light absorption in neural retina. A detailed analysis revealed that at a relative melanin concentration of 0.2 or larger, the melanin in the RPE and choroid layers absorbed most of the photons penetrating through the neural retina. However, retina light absorption decreased rapidly as the relative melanin concentration increased from 0 to 0.2 . Although the melanin concentration in choroid varies considerably among individuals, the RPE melanin concentration is independent of race [26,27]. Based on our simulation, the RPE layer in normal human RPE can absorb more than $75 \%$ of the total incident light, which is sufficient to prevent most backscattered photons from reentering the neural retina in different races. At wavelengths corresponding to high hemoglobin absorption, such as $550 \mathrm{~nm}$, albino neural retina absorption was at least $30 \%$ higher than that of pigmented retina. At $630 \mathrm{~nm}$, absorption in albino neural retina can be four times as large as that of the pigmented retina. In addition, neural retina absorption in humans was clearly subject to the effect of hemoglobin absorption at choroid when the relative melanin concentration was less than 0.2 .

\section{Absorption Spectra of Pigmented and Albino} Retinas

Figure 5 shows the absorption spectra in neural retina computed at different hemoglobin concentrations. In the figure legends, "A" refers to albino retina, "N" refers to normal retina, and the numbers indicate hemoglobin concentration in $\mathrm{mmol} / \mathrm{l}$. For example, "0.85A" indicates albino retina with a hemoglobin concentration of $0.85 \mathrm{mmol} / \mathrm{l}$.

In normal human retina, changing the hemoglobin concentration from 0.85 to $3.4 \mathrm{mmol} / \mathrm{l}$ had essentially no effect on neural retina absorption, and the curves completely overlapped. This was due to the fact that melanin absorption was dominant in RPE and choroid and overshadowed the effect of hemoglobin. However in albino subjects, hemoglobin concentration had a substantial impact on neural retina absorption because of the diminished attenuation from melanin. Such influence was wavelength dependent. Neural retina absorption increased when hemoglobin content decreased.

The absorption changes at neural retina caused by hemoglobin variations in choroid were attributed to the amount of photons backscattered from choroid and sclera. In order to clarify this, we separated the absorbed photons in neural retina into two categories: photons passing through the neural retina directly and photons backscattered into the neural retina. The results at three different

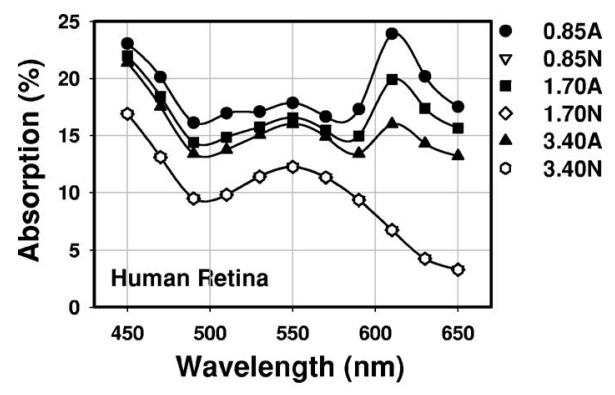

Fig. 5. Spectra of light absorption in albino and normal human neural retinas at different hemoglobin concentrations. 
wavelengths were shown in Table 3 . It can be seen that in normal pigmented eyes, the backscattered photons contributed less than $3 \%$ of the total absorbed photons. However, in the albino retina layer, the backscattered photons can be 3 times more than those directly passing.

Both choroid and sclera can contribute to backscattered photons. We isolated those backscattered from sclera in our simulation, and the results were plotted with the total backscattered light in Fig. 6 . It can be seen that the sclera contributed little in human neural retina absorption (about 0\%-30\% of total backscattered), which agrees well with the results of Preece and Claridge [10].

\section{E. Absorption Spectra in Dark-Adapted Retinas}

The above simulation results were calculated using retina optical properties measured in vitro [9], where photoreceptors were bleached. In this section, we show that light absorption in dark-adapted retina can be calculated by considering light absorption contributed from rod photoreceptors. The rod absorption coefficients [Fig. 7(a)] were calculated based on the optical density of rods measured by Liem et al. [28]. We assumed a neural retina layer with thickness of $200 \mu \mathrm{m}$ when converting the optical density to an optical absorption coefficient. Extrapolation was used to obtain the optical density at $650 \mathrm{~nm}$, and the value was close to zero. The optical absorption coefficients of dark-adapted neural retina were calculated as the summation of the absorption coefficients of the bleached neural retina and the rods. Although the large anisotropy of neural retina (Table 1) indicated highly forward scattering, the explicit waveguide effect of photoreceptors [29] was not considered in our calculation.

Figure 7(b) shows the comparison of the human neural retina absorption spectra under dark-adapted and bleached conditions. Blood concentrations were set as $1.7 \mathrm{mmol} / \mathrm{l}$. Because of the photoreceptor's contribution, the retina layer absorption under the dark-adapted condition was twice or even more than that under the bleaching condition. In addition, the melanin concentration has a slightly diminished effect on retina absorption in darkadapted retina than in bleached retina. Nevertheless, under dark adaptation, albino neural retina absorptions were still at least $\sim 20 \%$ higher than those of pigmented neural retina. Table 4 shows retinal light absorption at three different wavelengths $(510 \mathrm{~nm}, 550 \mathrm{~nm}$, and

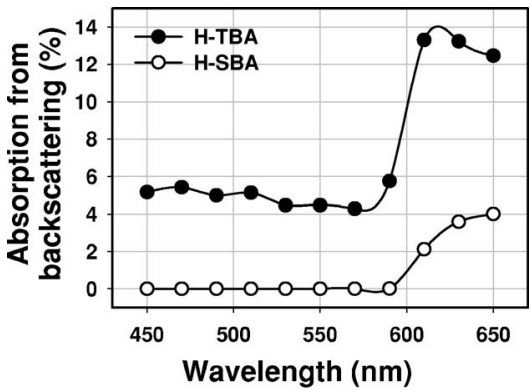

Fig. 6. Light absorption in albino human neural retina attributed to photons backscattered from sclera. H-TBA, total backscattered absorption; H-SBA, absorption due to backscattered photons from sclera. The hemoglobin concentration used in the simulation was $1.7 \mathrm{mmol} / \mathrm{l}$.

$630 \mathrm{~nm})$. Similar to bleached retina, the absorption of red light $(\sim 630 \mathrm{~nm})$ can be several times larger in albinism than in normal subjects because there is no contribution from rods at such wavelengths.

\section{F. Effect of Eye Size}

Because of the spherical model used, we expected that the eye size may have affected the photon propagation process. In order to verify this, we compared the photon absorption in mouse neural retina with that obtained in human neural retina.

Figure 8(a) indicates that the flat MCML model for mouse retina overestimated the neural retina layer absorption, which was exactly opposite to the case in humans (Fig. 3). In humans, the photons reentering the neural retina were the major contributors to the higher absorption in the spherical model. Due to a smaller diameter and thinner thickness of the choroid and sclera layers in mouse retina (Table 1), the incident photons could easily propagate into the fifth environmental layer. However, the flat model assumed that all the layers with fixed depth were always infinitely wide, which reduced a photon's chance of propagating into the environment and caused a higher absorption.

In a normal human or mouse, the absorption spectra were very similar to each other. At wavelengths smaller than $600 \mathrm{~nm}$, neural retina absorption increased when hemoglobin content decreased in both humans and mice. However, at wavelengths longer than $600 \mathrm{~nm}$, different

Table 3. Light Absorption in Bleached Human Neural Retina at Three Wavelengths ${ }^{a}$

\begin{tabular}{|c|c|c|c|c|c|}
\hline \multirow[b]{2}{*}{ Wavelength } & \multicolumn{3}{|c|}{ Absorption (\%) } & \multirow{2}{*}{$\begin{array}{c}\text { Backscatter/ } \\
\text { Direct }(\%)\end{array}$} & \multirow{2}{*}{$\begin{array}{c}\text { Total Albino/ } \\
\text { Pigmented }\end{array}$} \\
\hline & Direct & Backscatter & Total & & \\
\hline \multicolumn{6}{|l|}{$510 \mathrm{~nm}$} \\
\hline Pigmented & 9.71 & 0.09 & 9.80 & 0.93 & 1.51 \\
\hline Albino & 9.71 & 5.13 & 14.84 & 52.83 & \\
\hline \multicolumn{6}{|l|}{$550 \mathrm{~nm}$} \\
\hline Pigmented & 12.10 & 0.14 & 12.24 & 1.16 & 1.35 \\
\hline Albino & 12.09 & 4.47 & 16.56 & 36.97 & \\
\hline \multicolumn{6}{|l|}{$630 \mathrm{~nm}$} \\
\hline Pigmented & 4.15 & 0.10 & 4.25 & 2.41 & 4.09 \\
\hline Albino & 4.15 & 13.23 & 17.38 & 318.80 & \\
\hline
\end{tabular}

${ }^{a}$ The hemoglobin concentration used in the simulation was $1.7 \mathrm{mmol} / \mathrm{l}$. 

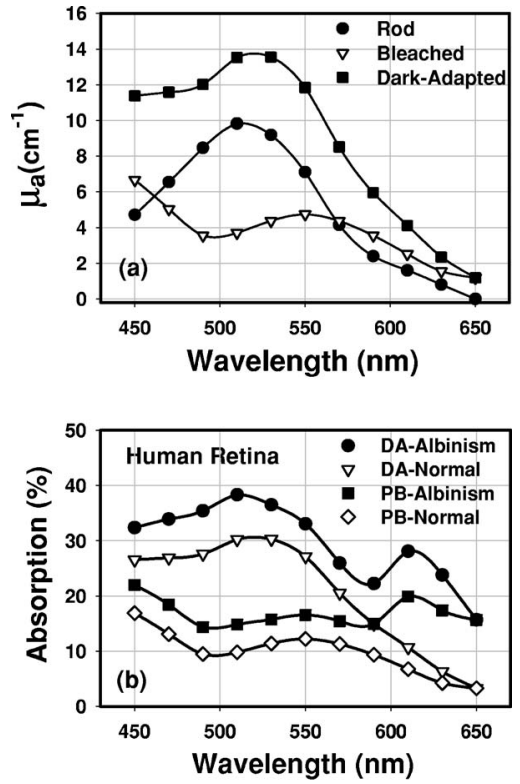

Fig. 7. (a) Optical absorption coefficients of rods and bleached and dark-adapted neural retinas. (b) Light absorption spectra in dark-adapted (DA) and bleached (PB) human neural retina.

results were obtained in human and mouse retinas. In the mouse, the hemoglobin concentration had no effect on mouse retinal layer absorption when light wavelengths were larger than $600 \mathrm{~nm}$. On the other hand, hemoglobin concentration showed a big influence on albino human retina absorption at such wavelengths. Such a difference can be explained also by considering the retina size difference. The choroid thickness in human retina was about $250 \mu \mathrm{m}$, and the sclera thickness was $700 \mu \mathrm{m}$. A significant amount of backscattered light from choroid and sclera was subjected to attenuation by hemoglobin. This was the reason that hemoglobin concentration had a big influence on human neural retina absorption at 600-650 nm, even though molar extinction coefficients of hemoglobin are very small at these wavelengths. In the mouse, the thickness of choroid was only $23 \mu \mathrm{m}$ and the sclera layer was only $30 \mu \mathrm{m}$ thick. Most of the photons passed through these two layers without being absorbed. Therefore, the variations in hemoglobin concentration at choroid had little impact on light absorption at neural
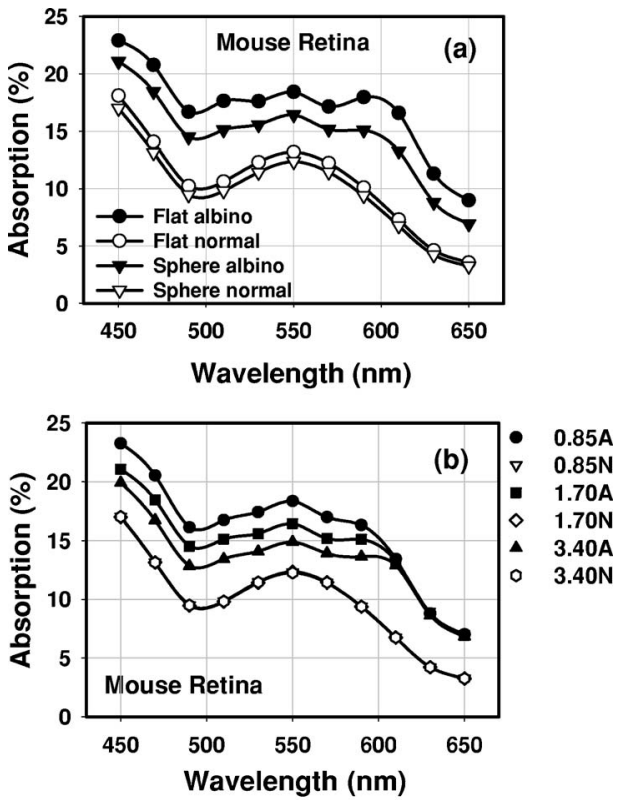

Fig. 8. (a) Light absorption in bleached mouse neural retina calculated by the spherical and slab retina models. The hemoglobin concentration used in the simulation was $1.7 \mathrm{mmol} / \mathrm{l}$. (b) Spectra of light absorption in albino and normal mouse neural retinas at different hemoglobin concentrations.

retina. Moreover, simulations indicated that mouse sclera scattered more photons (about $45 \%-60 \%$ of total backscattered) back into the neural retina because the mouse choroid layer is much thinner than that of a human.

\section{DISCUSSION}

It was observed that ERG signals in young albino animals increased significantly compared with those in young pigmented mice at all light intensities, but not in adult and aged mice [6]. Our results supported that the large increase in photon absorption in young albino retina must have contributed to the larger ERG amplitude observed. However, the adult and aged albino mice have suffered from photon damage during their lifetime, which leads to reduced ERG amplitudes.

A fundamental difference between direct-pass photons and backscattered photons is that the backscattered pho-

Table 4. Light Absorption in Dark-Adapted Human Neural Retina at Three Wavelengths ${ }^{a}$

\begin{tabular}{|c|c|c|c|c|c|}
\hline \multirow[b]{2}{*}{ Wavelength } & \multicolumn{3}{|c|}{ Absorption (\%) } & \multirow{2}{*}{$\begin{array}{c}\text { Backscatter/ } \\
\text { Direct }(\%)\end{array}$} & \multirow{2}{*}{$\begin{array}{c}\text { Total Albino/ } \\
\text { Pigmented }\end{array}$} \\
\hline & Direct & Backscatter & Total & & \\
\hline \multicolumn{6}{|l|}{$510 \mathrm{~nm}$} \\
\hline Pigmented & 30.10 & 0.15 & 30.25 & 0.50 & 1.27 \\
\hline Albino & 30.11 & 8.20 & 38.31 & 27.23 & \\
\hline \multicolumn{6}{|l|}{$550 \mathrm{~nm}$} \\
\hline Pigmented & 26.92 & 0.21 & 27.13 & 0.76 & 1.22 \\
\hline Albino & 26.93 & 6.12 & 33.05 & 22.71 & \\
\hline \multicolumn{6}{|l|}{$630 \mathrm{~nm}$} \\
\hline Pigmented & 6.17 & 0.14 & 6.31 & 2.30 & 3.77 \\
\hline Albino & 6.18 & 17.62 & 23.80 & 285.03 & \\
\hline
\end{tabular}

${ }^{a}$ The hemoglobin concentration used in the simulation was $1.7 \mathrm{mmol} / 1$. 

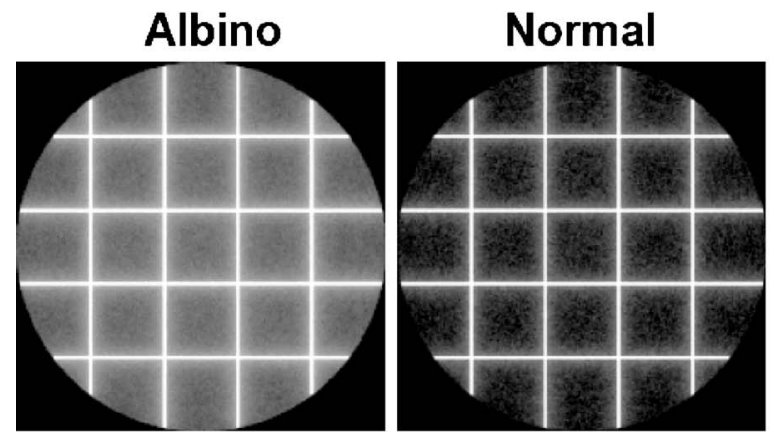

\section{$1 D$ absorption profile}

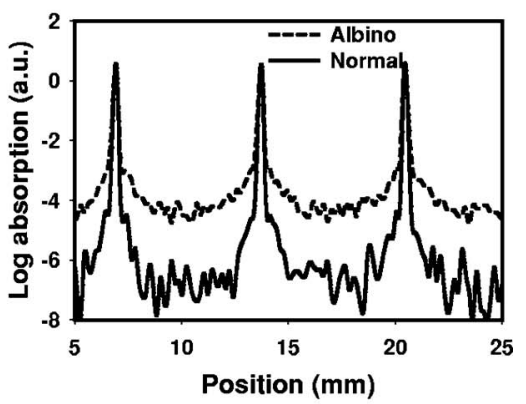

Fig. 9. Spatial distribution of light absorption in human neural retina. The retina was illuminated by a grid light source (510 nm). The image resolution for both was $0.17 \mathrm{~mm} /$ pixel. The $1 \mathrm{D}$ absorption profiles were obtained along a horizontal line across the absorption images. The hemoglobin concentration used in the simulation was $1.7 \mathrm{mmol} / \mathrm{l}$.

tons have random angular distributions. To illustrate such an effect, we computed images formed at albino and normal human neural retinas by projecting an incident light in grid patterns onto the retina surface. The calculated absorption images are shown in Fig. 9. In order to display the $3 \mathrm{D}$ profile as a $2 \mathrm{D}$ image, we stretched the retina layer along two orthogonal directions into a plane so that the two axes in Fig. 9 were the two orthogonal longitudes of the spherical retina layer. Therefore the original input grids were not rectangular at the retinal surface. The image size was $200 \times 200$ pixels $\left(34 \times 34 \mathrm{~mm}^{2}\right)$, and the image resolution for both was $0.17 \mathrm{~mm} /$ pixel. The gray scale of the picture represents the relative energy absorbed at each location of the retina layer. The bright color indicates higher absorption, and the dark color indicates lower absorption. It can be seen that the images obtained from albino retina were less sharp than those of normal ones. The background absorption at locations without incident light was much higher in albino retina than in pigmented retina. Such an effect was more significant in mouse than in human retinas because of the smaller mouse eye. The elevated backscattered light contributes to the vision deterioration in albino subjects. In addition, these results provided an explanation for the persistence of poor vision in albinos despite refractive corrections [30].

Because of the uncertainties regarding the absolute accuracies of the chosen optical properties, we studied the sensitivity of their effects on the calculation results. We found that anisotropy factor $g$ of the neural retina had the biggest effect on the calculated absorption. A $20 \%$ decrease of $g$ in neural retina increased absorption by $\sim 30 \%$ at $510 \mathrm{~nm}$ for a typical hemoglobin concentration of $1.7 \mathrm{mmol} / \mathrm{l}$. This was expected because of the broader scattering angle distribution associated with small $g$. The absorption coefficient also had a significant effect. A $20 \%$ change in the absorption coefficient in neural retina resulted in an $\sim 16 \%$ absorption change in the same layer. The variation of optical scattering coefficients had little effect on retina layer absorption. In addition, variations of optical coefficients at sclera and the outermost (fifth) layer had almost no influence on light absorption in neural retina.

Having discussed the importance of melanin concentration on light absorption in neural retina, it should be pointed out that light-induced retina damage could be as- sociated with various light-induced effects, such as mechanical, thermal, and photochemical effects [31]. The roles of melanin concentration on retinal photon damage may be different according to these different mechanisms. For example, Wasowicz et al. [32] showed that no apparent morphologic changes were found in retinas of pigmented rats after constant illumination, whereas in albino rats disappearances of photoreceptors and other cell bodies were observed. This result underscored the importance of the melanin concentration. However, in a different study, Gorgels and Norren [33] concluded that melanin did not play a crucial role in photochemical damage. Interestingly, melanin plays a significant antioxidant role, which is also related to retina light damage [34]. Nevertheless, light absorption is a critical step involved in light damage, and a quantitative light transport model in retina can provide valuable insight into the complicated problem of retina photon damage.

\section{CONCLUSION}

We have developed a Monte Carlo model to simulate the effects of melanin concentrations on light absorption in neural retina. Our results indicated that the spherical nature of the retinal structure must be considered in albino subjects. We showed that melanin concentrations at the RPE and choroid layers have a significant influence on neural retina absorption in both humans and mice. At visible wavelengths, the backscattered light from choroid and sclera contributed less than $3 \%$ of the total light absorption in pigmented retina. However, backscattered light contributed at least $20 \%$ of the total absorption in albino retina. At red wavelengths (such as $630 \mathrm{~nm}$ ) where hemoglobin absorption in choroid can be neglected, the backscattered light became the dominant contributor to retinal absorption. In addition, our results indicated that the retinal size difference between mice and humans had a significant effect on light absorption in albino subjects, which should be considered when extrapolating results obtained in different species. Our model can be applied to simulate retina absorption under any illumination conditions by incorporating proper photoreceptor absorptions. Such a quantitative Monte Carlo model may provide a useful tool in studying light-induced retina damage. 


\section{REFERENCES}

1. D. K. Sardar, F. S. Salinas, J. J. Perez, and A. T. Tsin, "Optical characterization of bovine retinal tissues," J. Biomed. Opt. 9, 624-631 (2004).

2. D. J. Creel, C. G. Summers, and R. A. King, "Visual anomalies associated with albinism," Ophthalmic Physiol. Opt. 11, 193-200 (1990).

3. R. Abadi and E. Pascal, "The recognition and management of albinism," Ophthalmic Physiol. Opt. 9, 3-15 (1989).

4. I. Russel-Eggitt, A. Kriss, and D. S. Taylor, "Albinism in childhood: a flash VEP and ERG study,” Br. J. Ophthamol. 74, 136-140 (1990).

5. A. J. Vingrys and B. V. Bui, "Development of postreceptoral function in pigmented and albino guinea pigs," Visual Neurosci. 18, 605-613 (2001).

6. J. Gresh, P. W. Goletz, R. K. Crouch, and B. Rohrer, "Structure-function analysis of rods and cones in juvenile, adult, and aged C57BL/6 and Balb/c mice," Visual Neurosci. 20, 211-220 (2003).

7. L.-H. Wang, S. L. Jacques, and L. Zheng, "MCML-Monte Carlo modeling of light transport in multi-layered tissues," Comput. Methods Programs Biomed. 47, 131-146 (1995).

8. G. Yao and M. A. Haidekker, "Transillumination optical tomography of tissue-engineered blood vessels: a Monte Carlo simulation," Appl. Opt. 44, 4265-4271 (2005).

9. M. Hammer, A. Roggan, D. Schweitzer, and G. Muller, "Optical properties of ocular fundus tissues-an in vitro study using the double-integrating-sphere technique and inverse Monte Carlo simulation," Phys. Med. Biol. 40, 963-978 (1995).

10. S. J. Preece and E. Claridge, "Monte Carlo modeling of the spectral reflectance of the human eye," Phys. Med. Biol. 47, 2863-2877 (2002).

11. B. L. Evans and S. B. Smith, "Analysis of esterification of retinoids in the retinal pigmented epithelium of the Mitf fit (vitiligo) mutant mouse," Mol. Vis 3, 11-22 (1997).

12. C. Schmucker and F. Schaeffel, "A paraxial schematic eye model for the growing C57BL/6 mouse," Vision Res. 44, 1857-1867 (2004).

13. M. Hammer, S. Leistritz, L. Leistritz, and D. Schweitzer, "Light paths in retinal vessel oxymetry," IEEE Trans. Biomed. Eng. 48, 592-598 (2001).

14. M. Hammer, D. Schweitzer, E. Thamm, and A. Kolb, "Noninvasive measurement of the concentration of melanin, xanthopyll, and hemoglobin in single fundus layers in vivo by fundus reflectometry," Int. Ophthalmol. 23, 279-289 (2001).

15. L. G. Henyey and J. L. Greenstein, "Diffuse radiation in the galaxy," Astrophys. J. 93, 70-83 (1941).

16. L. V. Wang and S. L. Jacques, "Source of error in calculation of optical diffuse reflectance from turbid media using diffuse theory," Comput. Methods Programs Biomed. 61, 163-170 (2000).

17. V. V. Tuchin, "Light scattering study of tissues," Phys. Usp. 40, 495-515 (1997).

18. J. M. Sandbach, P. E. Coscun, H. E. Grossniklaus, J. E. Kokoszka, N. J. Newman, and D. C. Wallace, "Ocular pathology in mitochondrial superoxide dismutase
(Sod2)-deficient mice," Invest. Ophthalmol. Visual Sci. 42, 2173-2178 (2001).

19. Y. Ming, P. V. Algvere, A. Odergren, L. Berglin, I. van der Ploeg, S. Seregard, and A. Kvanta, "Subthreshold transpupillary thermotherapy reduces experimental choroidal neovascularization in the mouse without collateral damage to the neural retina," Invest. Ophthalmol. Visual Sci. 45, 1969-1974 (2004).

20. S. Chakravarti, J. Paul, L. Roberts, I. Chervoneva, A. Oldberg, and D. E. Birk, "Ocular and scleral alterations in gene-targeted lumican-fibromodulin double-null mice," Invest. Ophthalmol. Visual Sci. 44, 2422-2432 (2003).

21. M. Hammer and D. Schweitzer, "Quantitative reflection spectroscopy at the human ocular fundus," Phys. Med. Biol. 47, 179-191 (2002).

22. J. Riesz, J. Gilmore, and P. Meredith, "Quantitative scattering of melanin solutions," Biophys. J. 90, 4137-4144 (2006).

23. Oregon Medical Laser Center (OMLG). "Optical properties spectra," http://omlc.ogi.edu/spectra/.

24. G. H. Daly, J. M. Dileonardo, N. R. Balkema, and G. W. Balkema, "The relationship between ambient lighting conditions, absolute dark-adapted thresholds, and rhodopsin in black and hypopigmented mice," Visual Neurosci. 21, 925-934 (2004).

25. G. W. Balkema and S. MacDonald, "Increased absolute light sensitivity in Himalayan mice with cold-induced ocular pigmentation," Visual Neurosci. 15, 841-849 (1998).

26. J. J. Weiter, F. C. Delori, G. Wing, and K. A. Fitch, "Retinal pigment epithelial lipofuscin and melanin and choroidal melanin in human eyes," Invest. Ophthalmol. Visual Sci. 27, 145-152 (1986).

27. C. N. Keilhauer and F. C. Delori, "Near-infrared autofluorescence imaging of the fundus: visualization of ocular melanin," Invest. Ophthalmol. Visual Sci. 47, 3556-3564 (2006).

28. A. T. Liem, J. E. Keunen, D. van Norren, and J. van de Kraats, "Rod densitometry in the aging human eye," Invest. Ophthalmol. Visual Sci. 32, 2676-2682 (1991).

29. B. Vohnsen, "Photoreceptor waveguides and effective retinal image quality," J. Opt. Soc. Am. A 24, 597-607 (2007).

30. R. V. Abadi, C. M. Dickinson, E. Pascal, and E. Papas, "Retinal image quality in albinos: a review," Ophthalmic Paediatr. Genet. 11, 171-176 (1990).

31. M. Boulton, M. Rozanowska, and B. Rozanowski, "Retinal photodamage," J. Photochem. Photobiol., B 64, 144-161 (2001).

32. M. Wasowicz, C. Morice, P. Ferrari, J. Callebert, and C. Versaux-Botteri, "Long-term effects of light damage on the retina of albino and pigmented rats," Invest. Ophthalmol. Visual Sci. 43, 813-820 (2002).

33. T. G. Gorgels and D. van Norren, "Two spectral types of retinal light damage occur in albino as well as in pigmented rat: no essential role for melanin," Exp. Eye Res. 66, 155-162 (1998).

34. Z. Wang, J. Dillon, and E. R. Gaillard, "Antioxidant properties of melanin in retinal pigment epithelial cells," Photochem. Photobiol. 82, 474-479 (2006). 